\title{
Management Of Sacral And Sacroiliac Joint Fractures By Percutaneous Screws
}

\author{
E.E.Ali, M.E.Al-AShab, M.I.kandil, S.A.Sholah and M.G.Fathi \\ Orthopedic Surgery, Dept., Faculty of Medicine, Benha Univ., Benha, Egypt \\ E-Mail: dr.mahmoud_gamal@yahoo.com
}

\begin{abstract}
Pelvic ring fractures are serious injuries which have been functionally determined by reduction quality; the objective of this research is to assess the effect of percutaneous fixation of the screw in the treatment of sacred fractures and sacroiliac joint damage. The research included 20 patients with sacral fractures and sacroilia joint injuries, with an average age of 32. Every single xray anteroposterior (AP), inlet and outlet views in the pelvis were radiologically evaluated for all patients. At the conclusion of the follow-up period, the outcomes were evaluated both clinically and radiologically. Overall Majeed outcomes were (clinically) judged to be acceptable among $16(80 \%)$ patients; eight (40\%) were excellent and eight (40\%) were good and only $4(20 \%)$ were unsatisfactory; $3(15 \%)$ were fair and 1 $(5 \%)$ were bad; Percutaneous sacroiliac pelvic fracture fixation has less damage, less bleeding, fast recovery, safe and effective minimally invasive operation technique. Percutaneous iliosacral vibrators are very demanding methods of placing highly scalable malpositions that are potentially associated with the risk of neurological damage or inadequate stability.
\end{abstract}

Keywords: Iliosacral screw, pelvic ring disruption, percutaneous.

\section{Introduction}

Pelvic Trauma is one of the most complex management in trauma care and occurs in 3 percent of skeletal injuries [1]. Unstable injuries account for 46 percent of pelvic trauma that result from high energy trauma and usually associated with other skeletal injuries [2]. Pelvic ring injuries are variable, difficult to treat and often associated with substantial morbidity and mortality [3]. Displaced unstable pelvic ring injuries are commonly associated with disruption of the osteoarticular junction of the sacroiliac joint, and stable fixation of sacroiliac joint is very important for stability of the pelvic ring [4].

Anatomically the pelvic ring is composed of three bones ( 2 innominate bones: ilium, ischium pubis and the sacrum). From anterior the pubic and the ischial rami are connected with symphysis pubis and posteriorly the sacrum and the 2 innominate bines are joined by sacroiliac joint. Pelvic stability depends on the posterior ligament structures, pelvic floor muscles and fascia [5].

The ligaments of the sacroiliac joint include the following: Anterior sacroiliac ligament, interosseous sacroiliac ligament, posterior sacroiliac ligament, sacrotuberous ligament, sacrospinous ligament [6].

The main function of the SI joint is to provide stability and attenuate forces to the lower extremities. The strong ligamentous system of the joint makes it better designed for stability and limits the amount of motion available [7].

Sacral fractures occur in approximately 45 percent of pelvic fractures. An associated neurologic injury of the lumbosacral plexus may occur in 25 percent of sacral fractures. Sacral fractures typically result from highenergy injuries, but there is increasing identification of low-energy insufficiency fractures of the sacrum and pelvis [8].

In the past, most of these fractures were managed conservatively or by open reduction internal fixation, these techniques were used traditionally and there were fraught with complications as most of them related to the wound like wound breakdown and pelvic hematoma due to extensile surgical approach, wound problems, bowel injury and incisional hernia. They were also associated with other complications including iatrogenic nerve injury and large volume blood loss, both primary and secondary [9].

Surgical fixation of unstable pelvic injuries provides improved fracture reduction, early weight bearing and mobilization, lower mortalities, shorter hospital stays, and superior functional outcomes compared to nonoperative treatment [10].

Closed reduction and percutaneous sacroiliac screw fixation of the posterior pelvic ring injuries was first described by Matta and then Routt. It has many advantages as compared to open techniques including minimal soft tissue disruption and limited blood loss, and lowered infection rates and these advantages make it increasingly popular $[11,12]$.

Percutaneous sacroiliac screw fixation of unstable pelvic fractures has less damage, less bleeding, pain light, quick recovery, which is safe and effective minimally invasive surgical technique [13]. 
However, this technique is not risk free, mainly due to anatomical considerations. The anatomical safe corridor which allows for the fixation of the ilium to the first and second sacral vertebrae can be narrow, limited by exiting nerve root anteriorly, neural foramina caudally and sacral canal posteriorly. Furthermore, the size and shape of the safe corridor is anatomically varied among individuals [14].

This technique is considered to be a highly demanding operative technique with a high rate of screw malposition, which may be associated with the risk of neurologic damage or inefficient stability [15]

\section{Patients and methods}

prospective study was done in Benha university hospitals, El-Bank El Ahly Trauma center hospital and Nasser institute between November 2019 and March 2021, It included 20 patients having sacral fractures and sacroiliac joint injuries, were included in this study. The average time from injury to surgical procedure was 6 days (range 3-15). Follow up period ranged from 6 to 12 months with an average of 9 months.

\subsection{The inclusion criteria}

Patients with displaced sacroiliac joint (SIJ) sacroiliac fractures, sacroiliac joint dislocation, sacral fractures, Recent Fractures, and dislocated joints less than 4 weeks since primary injury date age and Age above 18 years (skeletally mature patients).

\subsection{The exclusion criteria}

skeletally immature patients, hemodynamically unstable due to specific disease or any other causes which makes them unfit for surgery, Injuries older than 4 weeks, Active infection (local or systemic infection) and Open pelvic fractures.

\subsection{Clinical assessment}

Clinical scoring by Majeed score[16], This system is designed for the assessment of the pelvic injuries and is based on five clinical parameters which are :Pain (20 points) ,Work(20 points), Sitting (10 points), Sexual intercourse (4 points) and Standing (36 points) Need for walking aids (12 points) ,Gait unaided (12 points) and Walking(12 points), total score $=S U M$ (points for all 7 of the parameters), The higher the score the better the outcome.

\subsection{Radiographic evaluation}

Pre-operative AP radiograph, high quality CT pelvis ( $2 \mathrm{~mm}$ cuts) with $3 \mathrm{~d}$ reconstruction were done routinely, post-operative $\mathrm{x}$-rays within the first postoperative week and before the patients were discharged from the hospital. Follow up radiographs were taken at 6,12 weeks and six months postoperatively. The radiographs were assessed for fracture healing, vertical displacement, and the quality of reduction. The post. Reduction x-rays was graded according to Matta and Tornetta (17), into [fig. 1]: Excellent: residual vertical displacement less than $5 \mathrm{~mm}$. Good: residual vertical displacement from 5 to $10 \mathrm{~mm}$. Fair: residual vertical displacement 11-20 mm. Poor: residual vertical displacement more than 20 $\mathrm{mm}$.

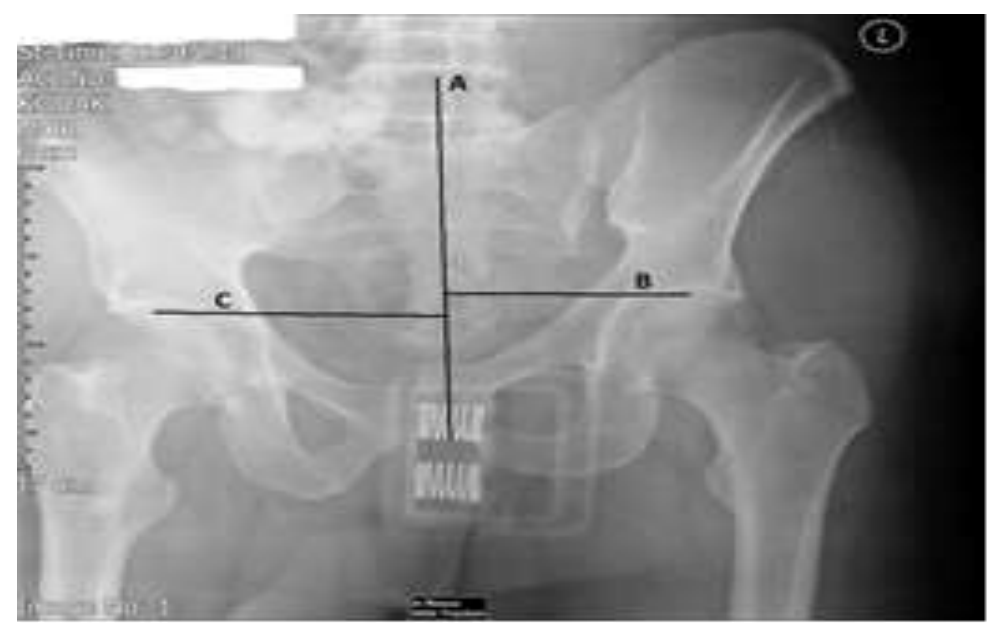

Fig. 1: A preoperative AP radiographs showing a Tile $\mathrm{C} 1$ pelvic injury. Line A represents a line passing through the axis of the sacrum. Lines B and C are drawn from the highest points of the femoral heads perpendicular to line A. The difference between the height of both femoral heads represents the vertical displacement of the affected hemipelvis.[17] 


\subsection{Surgical technique}

Once the patient's general condition permitted, the operation was done, an informed consent was taken from every patient, Position of the patient: supine on a radiolucent operating table, Reduction and fixation of the anterior arch was performed first, especially if the pubic symphysis was disrupted, This should improve posterior arch alignment, fixation could be performed by reconstruction plates or infix or external fixator, following steps were followed to insert the iliosacral screw (s) percutaneously:

- Lateral view radiograph, the ilio-cortical density which demarcates the anterior cortical thickening of the iliac part of the sacroiliac joint, it was determined on this view, it must be defined for secure entry point and the entry point of the guide wire was inferior to this mark as it should be below and behind the iliac cortical density and anterior to $\mathrm{S} 1$.

- Incision: a stab incision is made at the entry point explained before and the underlying tissues are dissected down to bone, by spreading with an appropriate blunt clamp, or with scissors if necessary.

- Insertion of the guide wire, the position is verified in true lateral, inlet, and outlet view. The guide wire is advanced about $1 \mathrm{~cm}$ into the sacral ala. The wire should be above S1 foramen in the outlet, anterior to the neural canal and not breaching the anterior sacral cortex in the inlet views.

- Position of screw according to its function: [fig. 2]

For SI joint fixation, the ISS is inserted perpendicular to the SI joint, and extends beyond the midline of the sacral body,
Compression screws are used for sacroiliac joint injuries.

For sacral fractures, the ISS is horizontal, allowing it to be inserted to or through the contralateral SI joint, to optimize fixation on both sides of the sacrum, fully threaded screws stabilize transforaminal sacral fractures.

- screw insertion: The screw length is measured with a ruler or gauge suitable for the guide wire, annulated screw $7.3 \mathrm{~mm}$ partially threaded screw is inserted with a washer. In case of comminution, fully threaded screws may be preferred to avoid over-compression of a sacral fracture.

- SI screw length for most adults, if oriented perpendicular to the joint surfaces, ranges from 70 to $90 \mathrm{~mm}$. Sacral fracture screws should be longer because the disorder is more medial than for SI joint injury and to achieve a balanced implant.

- A final check for the adequacy of the reduction and the screw position was done by intra-operative $x$-rays.

- Fixation of associated pelvic injuries, Reduction and fixation of the anterior arch was performed first, especially if the pubic symphysis was disrupted. This should improve posterior arch alignment, but ring alignment was not necessarily corrected. When a posterior injury could readily be reduced and fixed in anatomical alignment, this might be performed as a first step. This would provide a stable well-aligned hemipelvis upon which the rest of the reconstruction can be based.

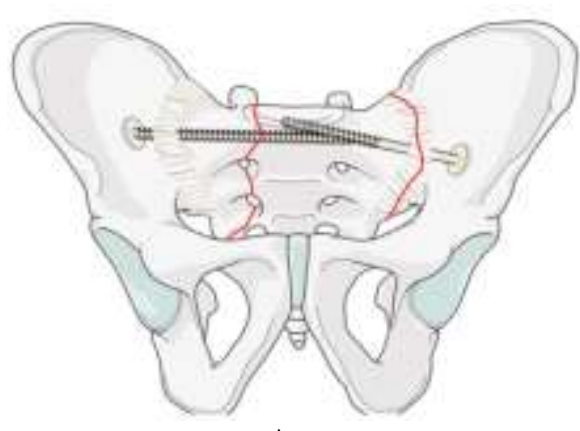

A

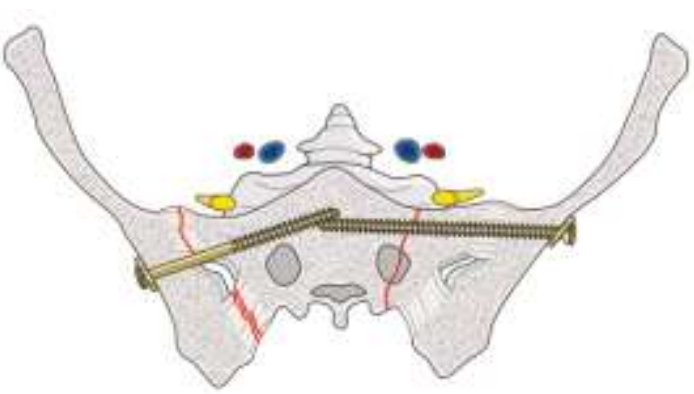

B

Fig. (2) A The screws are oriented according to the injuryand the desired function. The screws are inserted perpendicular to the injury; therefore, sacral, and iliosacral screws are different. Sacral screw orientation is more horizontal, which orients the screw perpendicular to the fracture and allows longer screw length to balance the fixation. iliosacral screws are oriented obliquely to remain perpendicular to the disrupted joint surfaces, B Compression screws are used for sacroiliac joint injuries, whereas fully threaded screws stabilize transforaminal sacral fractures [29]. 


\subsection{Complications and management}

Complications include injury to the superior gluteal artery, iliac vessels, and lumbosacral nerves, malreduction, malunion, nonunion, implant failure, and infection (Table 1). Damage to the superior gluteal artery may occur during the initial trauma or the procedure. Specifically, the deep superior branch of the superior gluteal artery is most at risk despite placing screws in the desired starting location.

The common and internal iliac veins lie on the ventral surface of the sacral ala at the level of S1, either anterior to the SI joint or immediately medial. Injury to these structures is serious and vascular or surgical consultation is warranted. Treatments include ligation or repair, retroperitoneal packing, and endovascular stent placement.

Neurologic injury may occur to the lumbosacral nerves and should be treated by screw revision., Malreduction and malunion are common complications, Infection rates after percutaneous SI screw insertion are very low.

\subsection{Postoperative care and follow up}

Recording of patients' operative data.

\section{Medications}

- antibiotics and thromboembolic prophylaxis against DVT and pulmonary embolus

- Appropriate analgesia for relieving the pain.

\section{Rehabilitation}

The patients were instructed to postpone weightbearing till 6 weeks. All patients, were advised to start toe touch weight bearing with crutches after 6 weeks and then partial weight bearing for another 6 weeks. Patients started full weight bearing at 12 weeks postoperatively.

Progressive weight bearing can begin according to anticipated healing. It should be remembered that pelvic fractures usually heal within 6-8 weeks, but that primarily ligamentous injuries may need longer protection (3-4 months).

The duration of follow-up ranged from 6 to 12 months with a mean of 9 months.

\section{Results}

From the analyzed 20 patients, $14(70 \%)$ were male and $6(30 \%)$ females; the average age of the patients was 32 (range, 18-60) years, the mechanism of injury was road traffic accident in 11 patients (55\%), fall from height in 9 patients $(45 \%)$, In the present study 14 patients were classified TILE C type (70\%) and 6 patients were classified TILE B type $(30 \%)$.

The results were assessed clinically according to Majeed score, table (2).

There was no statistical significance in the relation between Majeed score and the age, gender of the patients, The different modes of trauma, post-operative lag days and relation between Majeed score and TILE classification, whether type B or C. in this study.

Table (2) Distribution of the studied cases according to Majeed score $(n=20)$

\begin{tabular}{|c|c|c|c|c|c|c|c|c|c|}
\hline \multicolumn{2}{|l|}{ Score } & $\begin{array}{l}\text { Maximum } \\
\text { Score }\end{array}$ & Mean & Median & Range & $\begin{array}{c}\text { Median } \\
\text { Classification }\end{array}$ & \multicolumn{3}{|c|}{ Summary } \\
\hline \multicolumn{2}{|l|}{ pain } & 30 & 27 & 23 & $5-30$ & Slight/none & & No. & $\%$ \\
\hline \multicolumn{2}{|l|}{ work } & 20 & 8.6 & 6 & $0-20$ & $\begin{array}{l}\text { Light work/no } \\
\text { work }\end{array}$ & Unsatisfactory & 4 & 20.0 \\
\hline \multicolumn{2}{|l|}{ sitting } & 10 & 9.6 & 10 & $6-10$ & Pain free & \multirow[t]{2}{*}{ Poor } & \multirow[t]{2}{*}{1} & \multirow[t]{2}{*}{5.0} \\
\hline \multicolumn{2}{|c|}{ sexual intercourse } & 4 & 3.2 & 3 & $0-4$ & uncomfortable & & & \\
\hline \multirow[t]{3}{*}{ standing } & $\begin{array}{l}\text { Walking } \\
\text { aids }\end{array}$ & 12 & 10.7 & 12 & $4-12$ & No sticks & Fair & 3 & 15.0 \\
\hline & $\begin{array}{l}\text { Unaided } \\
\text { walk }\end{array}$ & 12 & 10.3 & 12 & $4-12$ & Normal & Satisfactory & 16 & 80.0 \\
\hline & $\begin{array}{l}\text { walking } \\
\text { distance }\end{array}$ & 12 & 9.1 & 10 & $4-12$ & $\begin{array}{l}1 \mathrm{~h} \text { without } \\
\text { sticks or limp }\end{array}$ & Good & 8 & 40.0 \\
\hline \multicolumn{2}{|l|}{ total } & 100 & 78.65 & 83.0 & $52.0-100.0$ & Good & Excellent & 8 & 40.0 \\
\hline
\end{tabular}

Relation between Majeed score and the working status of the patients in this study shows statistical significance with ${ }^{\mathrm{FE}} \mathrm{P}=0.013$, table (3). 
Table (3) Relation between Majeed score and working $(n=20)$.

\begin{tabular}{|c|c|c|c|c|c|c|}
\hline \multirow[t]{2}{*}{ Complications } & \multicolumn{2}{|c|}{$\begin{array}{l}\text { Majeed score } \\
\text { Unsatisfactory } \\
(n=4)\end{array}$} & \multicolumn{2}{|c|}{$\begin{array}{l}\text { Satisfactory } \\
(\mathbf{n}=16)\end{array}$} & $\chi^{2}$ & \multirow[t]{2}{*}{${ }^{\mathrm{FE}} \mathbf{p}$} \\
\hline & No. & $\%$ & No. & $\%$ & & \\
\hline No & 2 & 10.0 & 16 & 80.0 & $8.889^{*}$ & $0.032^{*}$ \\
\hline Yes & 2 & 10.0 & 0 & 0.0 & & \\
\hline
\end{tabular}

$\chi^{2}, \mathrm{p}: \chi^{2}$ and $\mathrm{p}$ values for Chi square test

FE: Fisher Exact for Chi square test *: Statistically significant at $\mathrm{p} \leq 0.05$

The ${ }^{\mathrm{FE}} \mathrm{P}$ was 0.032 regarding the relation between Majeed score and complications, showing statistical significance, table (4).

Table (4) Relation between Majeed score and complications $(\mathbf{n = 2 0})$

\begin{tabular}{|c|c|c|c|c|c|c|}
\hline \multirow[t]{2}{*}{ Workin } & \multicolumn{2}{|c|}{$\begin{array}{c}\text { Unsatisfactory } \\
(n=4)\end{array}$} & \multicolumn{2}{|c|}{$\begin{array}{c}\text { Satisfactory } \\
(n=16)\end{array}$} & \multirow[t]{2}{*}{$\chi^{2}$} & \multirow[t]{2}{*}{${ }^{\mathrm{FE}} \mathbf{p}$} \\
\hline & No. & $\%$ & No. & $\%$ & & \\
\hline No & 3 & 15.0 & 1 & 5.0 & $9.453^{*}$ & $0.013^{*}$ \\
\hline Yes & 1 & 5.0 & 15 & 75.0 & & \\
\hline
\end{tabular}

FE: Fisher Exact for Chi square test *: Statistically significant at $\mathrm{p} \leq 0.05$

Post-operative AP radiographs that assessed vertical displacement reduction according to Matta and Tornetta showed the following results:

- Nine patients were excellent with: residual vertical displacement less than $5 \mathrm{~mm}$, figure 38 \& figure 42.

- Eight patients were good with: residual vertical displacement from 5 to $10 \mathrm{~mm}$, figure 50.

- Three patients were fair with: residual vertical displacement $11-20 \mathrm{~mm}$.

Mean time for return to work was nine weeks mostly relevant to healing time in 16 patients as although seven patients had severe concomitant injuries. Sixteen of 20 patients were able to return to the same job without

modification. 3 changed their jobs because of posterior pelvic pain and 2, because of an associated injury to the hip.

Among the studied cases in this study no intra operative complication were detected. There were no iatrogenic vascular or neurologic injuries due to the screw osteosynthesis. All complications reported were postoperatively. Four patients reported superficial infection successfully treated by repeated dressing and systemic antibiotics.

Screw loosening developed in 1 patient after 10 weeks postoperatively; however, secondary displacement of the sacrum did not occur, only one patient had posterior pelvic pain improved with anti-inflammatory medications.
Mean time for healing was nine weeks, healing was assessed by clinical and radiological evaluation, fifteen patients ambulated normally. 2 had problems with ambulation attributable to pelvic pain and 7 from neurologic or associated injuries.

Eleven patients reported no pelvic pain and another 3 patients had pain only with strenuous activity. The other patients had pain with sitting or standing for long periods and with strenuous activity. No patient complained of pain or ambulatory deficiency due to pelvic obliquity.

\section{Discussion}

Iliosacral screw fixation is a wellrecognized technique for treating the unstable posterior pelvic lesion. Open method carries a high complication rate in association with impaired wound healing and the reported incidence was as high as 25 percent in one series [18]. These problems were avoided by the percutaneous methods. Closed reduction and percutaneous sacroiliac screw fixation of the posterior pelvic ring injuries was first described by Matta and then Routt.

Percutaneous iliosacral screw placement allows a minimally invasive fixation of posterior pelvic ring instabilities. The percutaneous iliosacral screw fixation procedure is performed using threedimensional fluoroscopy. It is very important to do a correct pelvis inlet and outlet X-ray projections.

In this study according to Matta and Tornetta [17] method of assessment of the quality of the reduction of the posterior pelvic 
injury, there were no patients in this study who had a poor radiological outcome. Only one patient in this group had a fair outcome and the other patients were classified as good or excellent. This was agreed with El-Desouky et al [2], who reported in their study which was done on 20 cases who were treated surgically by percutaneous iliosacral screw fixation technique and postoperative follow-up were evaluated radiologically according to the Matta and Tornetta [17]. Their grading was excellent in 14 cases, good in two cases $(5-10 \mathrm{~mm}$ displacement) and fair in four cases (10-20 mm displacement), and no poor cases were found. In comparison with plate and screws fixation, the radiological outcome was not statistically significant.

That was agreed with Lindahl et al [19] in their series of 101 surgically treated type C pelvic injury, who have used the same method of the radiological evaluation of the posterior displacement (as described by Matta and Tornetta [17]. Their final radiographic results were excellent in 66 patients, good in 25 patients, and fair in 10 patients. They also did not have poor results.

Patients' Clinical outcome can be assessed by multiple clinical scoring systems, in this study disease specific pelvic ring assessment tool was used, Majeed scoring system, which is one of the most commonly used scoring systems for the clinical assessment of patients with pelvic injuries [16]. The Majeed score has some advantages including assessment of the patient's level of pain and covering several functional aspects in the form of sitting, standing, walking and sexual impairment. In addition, the Majeed score, as being a specific patient reported outcome measure (PROM), has the advantage of involving patients in assessing their own level of disability and pain [20]. The disadvantages of the Majeed score include that neurological impairments, which have relevant prognostic influence, are not integrated into the score and as the other pelvic specific outcome scoring systems is not yet validated [21].

In this study, the overall Majeed score was $78.65 \pm 11.63$. The overall grading of the clinical outcome showed that 8 patients $(40.0$ percent ) had excellent, 8 patients (40.0 percent ) had good, 3 patients (15.0 percent ) had fair and only one patient (5.0 percent ) had poor clinical outcome. Thus, the patients with satisfactory (excellent and good) results were 16 patients (80.0 percent) which was similar to the results of El-Desouky et al [2], who reported in their study that the clinical scoring by Majeed score at the end of the follow-up period was excellent in seven cases, good in ten cases, fair in two cases (two cases with sacroiliac fracture dislocation with residual displacement between 10-20 mm) and poor in one patient, this was a case of internal hemipelvectomy with a traumatic neurological deficit that was recorded at the time of admission. Lindahl and Hirvensalo [22] who published 101 consecutive Tile classification type C pelvic fractures. All of their patients were treated surgically, with 78 patients receiving both anterior and posterior ring fixation. Their Majeed functional score results were excellent in 68 patients $(67.3$ percent ) good in 16 (15.8 percent ), fair in 16 (15.8 percent ) and poor in one patient (0.99 percent ) with 84 patients (83.2 percent ) obtained satisfactory results.

Mardanpour and Rahbar [23] analyzed 38 patients with unstable pelvic fractures, treated from 2002 to 2008 were retrospectively reviewed. Internal fixation was done by plate with ilioinguinal and kocher-langenbeek approaches for anterior, posterior pelvic wall and acetabulum fracture, respectively. Quality of reduction was graded according to Majeed score system. The functional outcome was excellent in 66 percent, good in 15 percent, fair in 11 percent and poor in 7 percent of the patients with type $B$ pelvic fractures and functional outcome was excellent in 46 percent , good in 27 percent, fair in 27 percent and poor in 0 percent of the patients with type $\mathrm{C}$ pelvic fractures. There were four postoperative infections. No sexual functional problem was reported. Neurologic problem like Lateral cutaneous nerve of thigh injury recovered completely in 2 patients and partially in 2 patients.

Another study (Chen et al) [24] was reported on a retrospective analysis of 32 patients with unstable pelvic ring injuries who were treated with percutaneous placement of iliosacral screws (group A of 15 patient) or conservative means (group B of 17 patient) from January 2002 to September 2009. Radiographic, clinical, and functional outcomes were compared between the two treatment groups. Patients who underwent percutaneous iliosacral screw fixation after pelvic trauma had better functional results than those treated conservatively, as per the Majeed grading system. Patients in group (A) also demonstrated less residual displacement on radiography at 1 year follow up than those in group (B). Finally, patients in group (A) had better pain relief at 1 month and 1 year follow ups than those in group (B), they also used the Majeed functional grading system, which allows easy and comprehensive assessment, including of specific problems caused by 
pelvic ring injury such as sitting or sexual intercourse. [14] The Majeed functional scores were better for group (A) than for group (B) on the pain $(\mathrm{p}=0.028)$, work $(\mathrm{p}=0.006)$, and sitting ( $\mathrm{p}=0.049)$ subscales; moreover, group (A) had a higher proportion of patients with grades of excellent and good as compared with group (B). The author concluded that Percutaneous iliosacral screw fixation for unstable posterior pelvic ring injuries results in less residual displacement at medium term follow up, and better pain relief at short and medium term follow up, than who received conservative treatment. Better functional outcomes were observed at 1 year follow up as compared with conservative treatment.

Another study done by Khaled et al [25] with the aim of assessing the clinical result and functional score of 43 patient of pelvic ring injury 20 patients go for ISSF iliosacral screw fixation, 22 go for plate fixation, and 1 patient lost. The average Majeed score for the group fixed with plates was 84.56 points (range: 66100 points), and it was lower than the Majeed score for ISSF, which was 87.2 points (range: 53-97 points). However, the difference was not statistically significant, with a P-value of 0 . 404. The author concluded that Percutaneous ISS fixation is a good option for fixation of post pelvic ring fractures, with lesser blood loss and shorter operative time compared with plate fixation. The functional outcome of the cases fixed with IS was better; however, the difference was not statistically significant.

Another study Khaled et al [26] for ISSF done on 77 cases (46 of tile $\mathrm{C}$ and 31 of Tile B) with postoperative follow up range from (615 months), they also used the Majeed scoring system for clinical evaluation, the clinical and radiological results were satisfactory as the clinical union occur in all cases and radiologically 55 patients were excellent 16 good and 6 fair with no poor results detected.

Another study done by El-badawy et al [27] on 12 patients of SIJ disruptions underwent surgery. Mode of injury was road traffic accident in 58.3 percent of cases and fall from height in 41.7 percent, According to tile classification Injuries of 5 patients (41.7 percent ) were classified B1and 2 patients (16.7 percent ) were B2. 5 patients (41.7 percent ) were $\mathrm{C} 2$, the duration of follow-up ranged from 6 to 12 months with a mean of 9 months. The patients were assessed both clinically and radiologically at the end of the follow-up period. Patients were subjected to clinical examination at the last follow up visit which was based on Majeed score. The final overall results were considered satisfactory in 9 (75 percent) patients; 3 (25 percent) were excellent, 6 (50 percent ) were good, and 3 patients (25 percent) were unsatisfactory fair outcome.

Another study done by Abou-Khalil S. et al [28] on 50 patients sustained an unstable posterior pelvic ring injury of these 50 patients, 36 adult patients were treated with iliosacral fixation. The patients were classified into two groups: the CRPF group had 22 patients stabilized under fluoroscopic (59 percent) or O-Arm guidance (41 percent ) and the ORIF group had 14 patients. One patient in the CRPF group and one patient in the ORIF group were lost to follow-up. Fourteen patients had to be excluded due to the following: patients under 18 years of age, patients whose initial posterior stabilization used a method other than CRPF and ORIF, H-shaped sacral fractures, and those treated only with external fixation. Fractures of the pelvis were classifed according to the AOTile classification. In the CRPF group, 19 patients had a Tile $\mathrm{C}$ and 3 patients a Tile $\mathrm{B}$ fracture. In the ORIF group, 8 patients were classifed according to Tile $\mathrm{C}$ and 6 according to Tile B .

Based on the Majeed's grading score for pelvic fractures, they had better functional outcomes using the CRPF technique with a Majeed's median score of 87 points compared to 69 points for the ORIF technique. We found 71 percent of good to excellent results with the CRPF group versus 46 percent with the ORIF group (119)

\section{Conclusion}

Cutaneous Iliosacral tissue screws may be utilized to anchor later pelvic ring volatility in safety, particularly in the context of severe soft tissue damage. Adequate intra-operating fluoroscopic views reduce neurovascular damage and malreduction, minimal risk of infection, and fewer difficulties with wound healing.

\section{References}

[1] C. Arvieux, F. Thony and C.Broux Current management of sever pelvic and perineal trauma. $\mathrm{J}$ Visc Surg.vol.149,pp. 227-38,2012.

[2] E.EL-Desouky, M.Mohamed and A.kandil Percutaneous iliosacral screw fixation in vertically unstable pelvic injuries, a refined conventional method, Acta Orthop Belg.vol.82,pp.52-59, 2016.

[3] T.Manson, RV.O'Toole, A.Whitney Young-Burgess classification of pelvic fractures does it predict mortality, transfusion requirements, 
and non-orthopaedic injuries? Orthop Trauma.vol.24,pp.603-9,2010.

[4] W.Choy, K.Kim, S.Lee and H.Park Anterior pelvic plating and sacroiliac joint fixation in unstable pelvic ring injuries Yonsei Med J.vol.53(2),pp.422-426, 2012.

[5] W.Kim Treatment of unstable pelvic ring injuries Journal List Hip PELVIS.vol.26(2),pp.79-83,2014.

[6] A.Vleeming, M. D.Schuenke, A. T.Masi , J. E.Carreiro, L.Danneels , F. H.Willard."The sacroiliac joint An overview of its anatomy function and potential clinical implications Journal of Anatomy.vol.221 (6),pp. 53767,2012.

[7] SP.Cohen Sacroiliac Joint Pain A Comprehensive review of anatomy diagnosis and treatment Anesth Analg.vol.101,pp.1440-1453,2005.

[8] S.Mehta, JD.Auerbach, CT.Born, KR.Chin Sacral fractures. J Amer Acad Orthop Surg.vol. 14,pp.656$665,2006$.

[9] R.Luukkainen, PV.Wennerstrand, HH.Kautiainen Efficacy of periarticular corticosteroid treatment of the sacroiliac joint in nonspondyloarthropathic patients with chronic low back pain in the region of the sacroiliac joint Clin Exp Rheumatol.vol.20,pp.52-4,2002.

[10]JR.Langford, AR.Burgess, Fa.Liporace Pelvic fractures. Part 2 Contemporary indications and techniques for definitive surgical management. J Am Acad Orhtop Surg.vol. 21,pp.458-68, 2013.

[11]J.Matta, T.Saucedo Internal fixation of pelvic ring fractures Clin Orthop Relat Res.vol.242,pp.83-97, 1989.

[12]ML Jr.Routt, PJ.Kregor, PT.Simonian Early results of percutaneous iliosacral screws placed with the patient in the supine position. $\mathrm{J}$ Orthop Trauma.vol.9,pp.207214,1995.

[13]Y.Krishnan Sharma and G.Magdum A retrospective analysis of percutaneous S1 joint fixation in unstable pelvic fractures Our experience in armed forces Med J Armed Forces India.vol. 72(3),pp.231-235,2016.

[14]AN.Miller, ML Jr.Routt Variations in sacral morphology and implications for iliosacral screw fixation. J Am Acad Orthop Surg.vol.20,pp.816,2012 .
[15]J.Zwingmann, G.Konrad, AT.Mehlhorn, NP.Südkamp , Oberst M Percutaneous iliosacral screw insertion: malpositioning and revision rate of screws with regards to application technique (navigated vs. Conventional). Trauma.vol.69,pp.1501-1506,2010.

[16]SA.Majeed,Grading the outcome of pelvic fractures. J Bone Joint Surg Br.vol.71(2),pp.304-306,1989.

[17]JM.Matta , and P.Tornetta, Internal fixation of unstable pelvic ring injuries. Clin Orthop Relat Res.vol. 329,pp.129-140,1996.

[18]JF.Kellam, RY.McMurtry, D.Paley . The unstable pelvic fracture. Operative treatment. Orthop Clin North Am.vol.18,pp. 25-41,1987.

[19]J.Lindahl and E.Hirvensalo, Outcome of operatively treated type-C injuries of the pelvic ring. Acta Orthop.vol.76(5),pp.667-678,2005.

[20]JF.Keating, J.Werier, P.Blachut. Early fixation of the vertically unstable pelvis: the role of iliosacral screw fixation of the posterior lesion. J Orthop Trauma.vol.13,pp.107$13,1999$.

[21]M.Tile, D.Helfet, J.Kellam. Fractures of pelvis and acetabulum. New YorkThieme.vol.55,pp.83,2015.

[22]J.Lindahl and E.Hirvensalo Outcome of operatively treated type-C injuries of the pelvic ring. Acta Orthop.vol.76(5),pp.667-678,2005.

[23]K.Mardanpour and M.Rahbar,The outcome of surgically treated traumatic unstable pelvic fractures by open reduction and internal fixation. $\mathrm{J}$ Inj Violence Res.vol.5(2),pp.7783,2013.

[24]P.Chen, W.Hsu, Y.Li . Outcome analysis of unstable posterior ring injury of the pelvis: Comparison between percutaneous iliosacral screw fixation and conservative treatment. Biomed J.vol.36,pp.289-294,2013.

[25]S.Khaled, M.Abdel Karim, A.Abdel Azeem , Management of cresent fractures-islocation of the sacroiliac joint:iliosacral screws versus plate fixation.The Egyptian Ortopedic Journal.vol.23,pp.123,2016.

[26]] SA.Khaled, O.Soliman and MA.Wahed, Functional outcome of unstable pelvic ring injuries after iliosacral screw fixation: single versus two screw fixation. Eur $\mathbf{J}$ Trauma 
Emerg Surg.vol.41(4),pp.387$392,2015$.

[27]El-badawy, Mohamed El-sayed. "Outcome of Percutaneous Iliosacral Screw Fixation of Sacroiliac Joint Disruptions." The Egyptian Journal of Hospital Medicine.vol.78.2,pp.234239,2020.

[28]S.Abou-Khalil, S.Steinmetz, L.Mustaki, B.Leger, E.Thein, O.Borens, Results of open reduction internal fixation versus percutaneous iliosacral screw fixation for unstable pelvic ring injuries: retrospective study of 36 patients. European Journal of Orthopaedic Surgery \& Traumatology.vol.45,pp.147,2020.

[29]Tile, Marvin, David L Helfet, Mark Vrahas, and James F Kellam: Fractures of the Pelvis and Acetabulum: Principles and Methods of Management Fourth edi. Thieme.vol.21,pp.74,2015 . 